\title{
Usefulness of indirect open reduction via a transconjunctival approach for the treatment of nasal bone fracture associated with orbital blowout fracture
}

Tae Ho Kim,

Seok Joo Kang,

Seong Pin Jeon,

ji Young Yun,

Hook Sun

Department of Plastic and Reconstructive Surgery, Inje University Busan Paik Hospital, Inje University School of Medicine, Busan, Korea

\begin{abstract}
Background: Nasal fracture and orbital blowout fracture often occur concurrently in cases of midface blunt trauma. Generally, these multiple fractures treatment is surgery, and typically, the nasal bone and orbit are operated on separately. However, we have found that utilizing a transconjunctival approach in patients with concurrent nasal bone fracture and orbital blowout fracture is a useful method.

Methods: The participants in the present study included 33 patients who visited the Plastic Surgery outpatient department between March 2014 and March 2017 and underwent surgery for nasal fracture and orbital blowout fracture. We assessed patients' and doctors' satisfaction with surgical outcomes after indirect open reduction via a transconjunctival approach for the treatment of nasal bone fracture with associated orbital blowout fracture.

Results: According to the satisfaction scores, both patients and doctors were satisfied with transconjunctival approach.

Conclusion: We presented here that our method enables simultaneous operation of nasal fracture accompanied by orbital blowout fracture, rather than treating the two fractures separately, and it allows precise reduction of the nasal fracture by direct visualization of the fracture site without any additional incisions or difficult surgical techniques. Also, by preventing the use of excessive force during reduction, this method can minimize damage to the nasal mucosa, thereby reducing the incidence of nasal bleeding.
\end{abstract}

Keywords: Nasal bone / Orbital fractures / Facial bones

\section{INTRODUCTION}

\begin{abstract}
Nasal fracture and orbital blowout fracture can often occur concurrently in cases of blunt trauma to the midface. The treatment of choice for these multiple fractures is surgery, and typically, the nasal bone and orbit undergo separate operations. Orbital fractures require surgery using a subciliary or transconjunctival approach, while nasal fractures are treated by closed or open reduction.
\end{abstract}

\section{Correspondence: Seok Joo Kang}

Department of Plastic and Reconstructive Surgery, Inje University Busan Paik Hospital, Inje University School of Medicine, 75 Bokji-ro, Busanjin-gu, Busan 47392, Korea

E-mail: sonydr@naver.com

Received February 21, 2018 / Revised April 19, 2018 / Accepted April 26, 2018
Closed reduction of the nasal bone is a relatively short and simple form of surgery that does not leave a scar. However, there is a higher risk of nasal deformity because the reduction is less precise. Furthermore, outcomes can differ depending on the surgeon's aptitude, and there are potential adverse effects involved with this technique such as nasal bleeding or adhesion of the nasal mucosa. Conversely, open reduction via skin incision has the advantage of providing better visibility to enable a more precise reduction. However, because it results in a postoperative scar, open reduction is only performed selectively in cases of open fracture.

Various techniques have been introduced in order to overcome the respective limitations of closed and open reduction, including 
indirect open reduction through an intercartilaginous incision, an endonasal incisional approach and endoscopic reduction [1-5]. We have obtained precise and satisfactory surgical outcomes in patients with concurrent nasal fracture and orbital blowout fracture by using a transconjunctival incisional approach and simultaneously reducing both fractures without any further incision. In this paper, we describe our use of this surgical method and the results we have obtained thus far.

\section{METHODS}

\section{Patients}

The participants in the present study included 33 patients who visited the Plastic Surgery outpatient department from March 2014 to March 2017 and underwent surgery for nasal fracture and orbital blowout fracture. All operations were performed by a single specialist (S.J.K) in plastic surgery. In total, there was 19 male patients and 14 female patients whose ages ranged from 8 to 67 years (mean, 30.5 years) (Table 1 ).

Preoperatively, all patients were photographed in a seated position. After 6 postoperative months, the patients visited the outpatient department to be photographed again in the same position and to complete a questionnaire. After 1 postoperative year, patients visited one more time and completed a questionnaire relating to any uncomfortable symptoms. Non-enhanced facial bone computed tomography (CT) and radiography in the Water view and nasal bone view, laterally, and from both sides, were performed pre- and postoperatively.

Table 1. Patient demographics

\begin{tabular}{lccc} 
Age $(\mathrm{yr})$ & Male & Female & No. of patients \\
$<10$ & 1 & 0 & 1 \\
\hline $10-19$ & 3 & 3 & 6 \\
\hline $20-29$ & 4 & 5 & 9 \\
\hline $30-39$ & 6 & 4 & 10 \\
\hline $40-49$ & 3 & 1 & 4 \\
$50-59$ & 1 & 1 & 2 \\
$\geq 60$ & 1 & 0 & 1 \\
\hline Total & 19 & 14 & 33 \\
\hline
\end{tabular}

\section{Surgical technique}

All surgeries were performed under general anesthesia. First, 0.3 $\mathrm{mL}$ of $2 \%$ lidocaine hydrochloride hydrate, mixed with 1:100,000 epinephrine, was injected into the medial bulbar conjunctiva for the purpose of hemostasis. We then conducted a forced traction test to evaluate passive eye movement. Thereafter, a 10- to $12-\mathrm{mm}$ incision was made in the conjunctiva using a No. 15 blade, including a 1- to 2-mm incision on the medial side of the punctum. The bone was then exposed by dissecting along the orbital septum up to the arcus marginalis. Next, a periosteal elevator was used to lift the periosteum in the direction of the frontonasal process of the maxilla to expose the fractured lateral nasal wall (Fig. 1).

Closed reduction of the nasal bone was then achieved by applying manual pressure, or using Walsham's forceps inserted through the nares, depending on the state of the nasal fracture. In cases which also involved septal fracture, we inserted Ash forceps into the nares, the fracture was reduced, and an external splint was applied for fixation.

When performing open nasal bone reduction using a transconjunctival approach, complete reduction was checked visually via the transconjunctival incision, and closed reduction of the nasal fracture was performed (Fig. 2). A Medpor Titan 0.85-mm Barrier sheet (Stryker, Kalamazoo, MI, USA) was inserted to cover the blow out fracture site. Next, a long, curved periosteal elevator was used to check the stability of the posterior margin of the or-

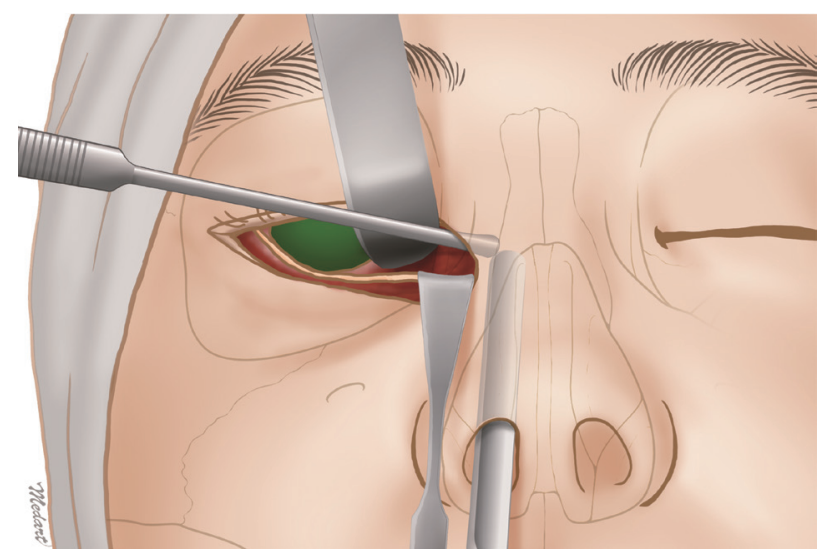

Fig. 1. Illustration of an indirect open reduction via a transconjuncti$\mathrm{val}$ approach. A periosteal elevator was used to lift the periosteum in the direction of the frontonasal process of the maxilla to expose the fractured lateral nasal wall. 

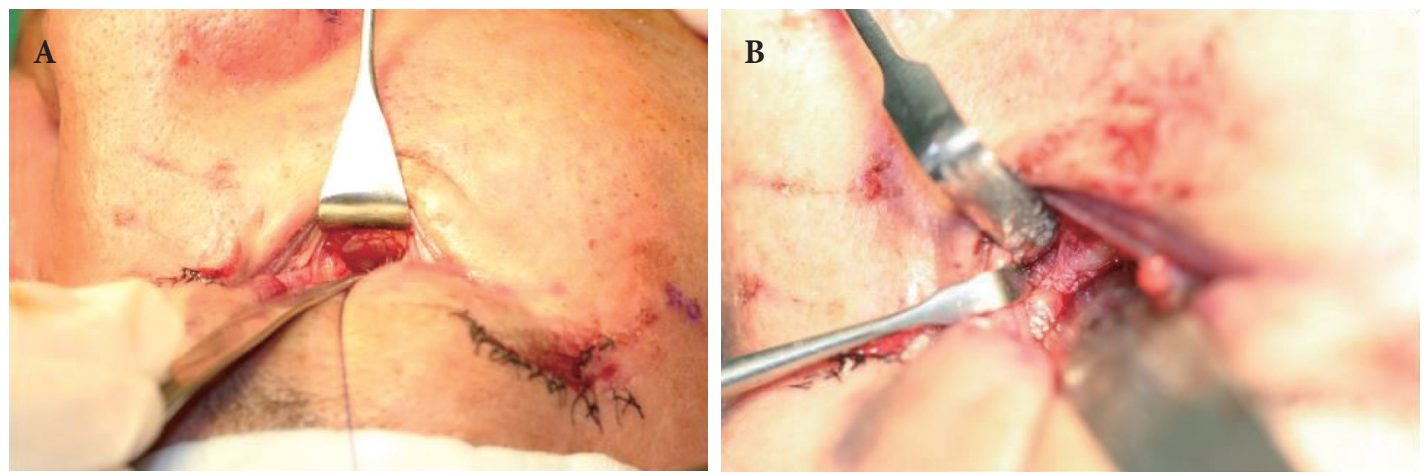

Fig. 2. Images of an indirect open reduction via a transconjunctival approach. (A) Following a conjunctival incision, the bone was exposed by dissecting along the orbital septum up to the arcus marginalis. (B) A periosteal elevator was used to lift the periosteum in the direction of the frontonasal process of the maxilla to expose the fractured lateral nasal wall.

bital wall, and Vicryl 6-0 sutures were placed in the incision site, layer by layer. Finally, intranasal petrolatum gauze or Merocel sponge (Medtronic Xomed, Jacksonville, FL, USA) packing was performed, and an external splint was applied for fixation.

On the fifth postoperative day, the intranasal petrolatum or Merocel sponge packing was removed, and on the seventh postoperative day, the patient was discharged if they had no discomfort. Patients visited the outpatient department for follow-up 1 month later.

\section{Assessment}

Six months after surgery, patients and physicians completed a questionnaire in which all items were answered as "very satisfied," "satisfied," "fair," "dissatisfied," or "very dissatisfied." Patient satisfaction was assessed for postoperative nasal congestion, discomfort (such as difficulty breathing through the nose), and visible postoperative depression or deviation (Table 2). Postoperative outcomes were also assessed by two specialists in plastic surgery (H.S., S.P.J.) who were not involved in the operation. By comparing pre- and postoperative photographs, facial bone CT scans, and radiographs taken during outpatient visits, physicians evaluated the precision of fracture reduction, and the deviation and symmetry of the nose (Table 2).

One year after surgery, patients visited the outpatient department again and completed a questionnaire relating to discomfort including "nasal hump," "nasal widening," "deviation," "nasal airway obstruction," and "hyposmia."

\section{RESULTS}

In terms of age, the most common age group was 30 to 39 years (10 out of 33 patients, 30.3\%). Six patients (18.1\%) were aged 10 to

Table 2. Patient and surgeon satisfaction 6 months after surgery

\begin{tabular}{|c|c|c|c|c|c|}
\hline Satisfaction scale & Very satisfied & Satisfied & Fair & Dissatisfied & Very dissatisfied \\
\hline \multicolumn{6}{|l|}{ Patient } \\
\hline Nasal congestion & 16 & 8 & 5 & 3 & 1 \\
\hline Nasal bleeding & 18 & 6 & 5 & 2 & 2 \\
\hline Trouble breathing & 23 & 5 & 2 & 2 & 1 \\
\hline Depression & 17 & 7 & 5 & 4 & 0 \\
\hline Deviation & 21 & 7 & 3 & 2 & 0 \\
\hline Overall severity & 20 & 8 & 3 & 1 & 1 \\
\hline \multicolumn{6}{|l|}{ Surgeon } \\
\hline Deviation & 19 & 10 & 2 & 1 & 1 \\
\hline Asymmetry & 21 & 7 & 3 & 2 & 0 \\
\hline Irregularity & 20 & 8 & 2 & 2 & 1 \\
\hline Overall improvement & 21 & 9 & 2 & 1 & 0 \\
\hline
\end{tabular}


19 years, nine (27.2\%) were aged 20 to 29 years, four $(12.0 \%)$ were aged 40 to 49 years, two (6.0\%) were aged 50 to 59 years, one $(3.0 \%)$ was aged $<10$ years, and one $(3.0 \%)$ was aged $\geq 60$ years. The ratio of male-to-female patients was 1.4:1, meaning that there were more male patients (Table 1). Based on interviews conducted at each patient's initial visit, there were five different causes of injury (fall, slip and fall, blunt trauma, human violence, and traffic accident), of which human violence was the most common cause, observed in 19 of the 33 patients (57.6\%) (Table 3).

In the patient satisfaction survey, the results were very satisfactory in 20 patients, satisfactory in eight, fair in three, dissatisfactory in one, and very dissatisfactory in one. Reasons for dissatisfaction were mostly deviation and nasal congestion (Table 2). In the questionnaire completed by two plastic surgery specialists, photographs, facial bone CT scans, and radiographs were compared (Figs. 3-5). The results were very satisfactory in 21 patients, satisfactory in nine, fair in two, dissatisfactory in one, and very dissatisfactory in no patients (Table 2). In the patient with a dissatisfactory outcome, physicians identified deviation and imprecise reduction.

One year after surgery, patients visited the outpatient department again and completed a questionnaire relating to uncomfortable symptoms. There was one patient who complained of nasal hump, one patient who complained of deviation and one patient who complained of nasal airway obstruction. None of the patients complained of nasal widening or hyposmia. In total, three of the $33 \mathrm{pa}-$ tients complained of discomfort after 1 postoperative year (Table 4).

\section{DISCUSSION}

As the farthest protruding part of the face, the nose is easily injured, and even a small deformity of the nasal bone or soft tissue

Table 3. Causes of injury

\begin{tabular}{lc} 
Cause & No. of patients \\
Fall & 2 \\
Slip and fall & 3 \\
\hline Blunt trauma & 3 \\
Human violence & 19 \\
Traffic accident & 6 \\
\hline Total & 33 \\
\hline
\end{tabular}
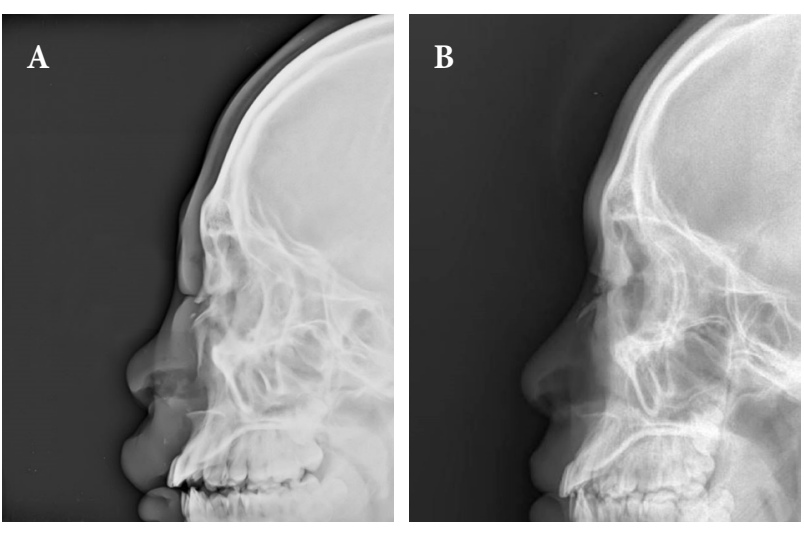

Fig. 3. A case involving depressed lateral nasal bone fracture. Preoperative (A) and postoperative (B) facial bone computed tomography view of the depressed right lateral nasal bone fracture.
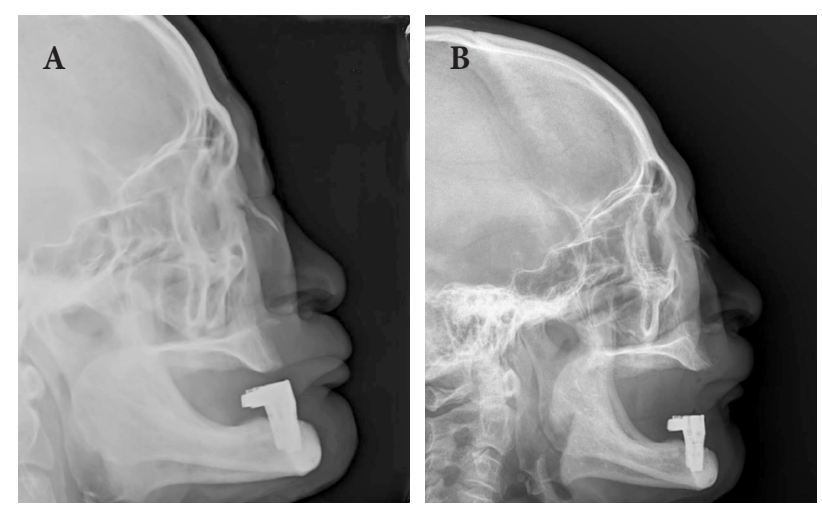

Fig. 4. A case involving depressed lateral nasal bone fracture. Preoperative (A) and postoperative (B) facial bone computed tomography view of the depressed left lateral nasal bone fracture.

Table 4. Complications after 1 year

\begin{tabular}{lc} 
Complication & No. of patients \\
\hline Nasal hump & 1 \\
Nasal widening & 0 \\
\hline Deviation & 1 \\
Nasal airway obstruction & 1 \\
\hline Hyposmia & 0 \\
\hline Total & 3 \\
\hline
\end{tabular}

can be easily seen. After facial injury, nasal deformities are particularly visible because the nose is at the center of the face, there is not enough strong muscle around the nasal bone to fill the fracture site, and the very thin skin and soft tissue covering the protruding part of the nose is anatomically distributed only over the most vulnerable areas. 

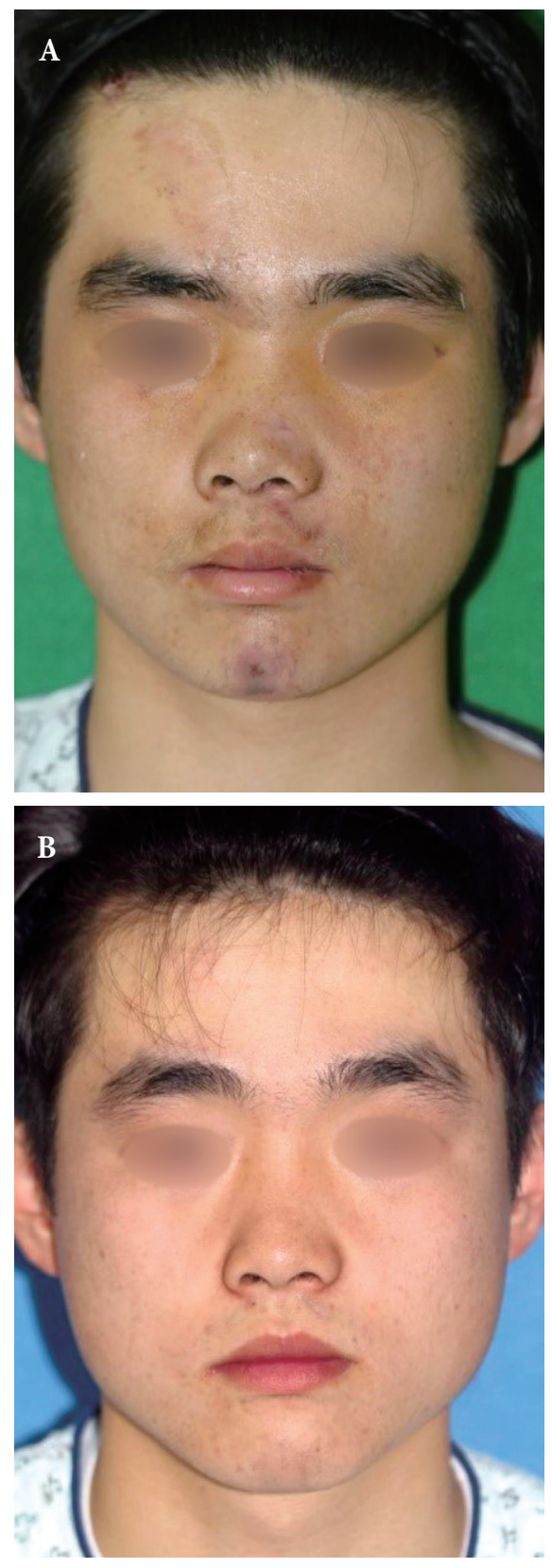

Fig. 5. A case involving nasal bone fracture and orbital blowout facture. (A) Preoperative photograph. (B) The photograph taken six months after surgery showing a satisfactory result.

Treatment for nasal fractures can traditionally be divided into closed and open reduction. Closed reduction is a relatively simple and easy procedure to perform under local or general anesthesia $[5,6]$. For this reason, a number of surgeons choose closed reduction although many studies have reported postoperative limitations related to this technique. Rohrich and Adams [1] reported that following closed reduction, a large proportion of patients showed nasal deformity and expressed dissatisfaction, and that approximately $50 \%$ of patients required re-surgery or further rhinoplasty. Moreover, unnecessary closed reduction can cause damage to the nasal mucosa, potentially resulting in nosebleeds after recovery due to mucosal adhesion.

In one study, a significant proportion (47.3\%, 185 out of 391 patients) of all blowout fracture patients had a complex fracture involving the nasal and orbital bones and a significant proportion of patients (6.4\%, 25 out of 391 patients) had concurrent nasal bone and orbital medial wall fractures [7]. Therefore, in the present study, we utilized a transconjunctival approach in patients with concurrent nasal bone fracture and orbital blowout fracture. We achieved efficient closed reduction of nasal fractures by performing dissection during the orbital fracture surgery to approach the lateral nasal wall and acquire a direct view of the fracture site [8]. Compared with conventional closed reduction, this procedure enables more precise reduction because the fracture site is visible during the procedure. Even compared with indirect open reduction, a technique introduced by Burm and Oh [4] in 1998, our procedure can be considered more precise because the fracture site can be observed with the naked eye. Moreover, compared to a previous study in which a subciliary incision was used to reduce nasal bone fractures and zygomatic arch fractures in a group of patients with blowout fracture, although the direct view of the fractured site was the same for the transconjunctival approach, meticulous dissection by an experienced surgeon resulted in lower risks of incision scar and postoperative ectropion or scleral show for the transconjunctival approach, which can be considered major advantages $[9,10]$.

These outcomes were also verified based on the postoperative satisfaction of patients and assessment by physicians. Approximately $85 \%$ of patients were "satisfied" or "very satisfied" in terms of improvements in nasal congestion, nasal bleeding, and breathing through the nasal cavity compared with preoperative levels, as well as nasal symmetry and overall satisfaction. Moreover, in our study, compression was maintained for 1 postoperative month using a nasal bone external splint, in order to prevent the tent effect, which can occur in severe nasoethmoidal orbital fractures. 
As a result of this practice, none of the patients in our study experienced this postoperative complication. Plastic surgery specialists also evaluated surgical outcomes by comparing pre- and postoperative photographs, radiographs and CT scans. These specialists reported that, compared with preoperative images, patients showed overall improvement in the external appearance of the nose, including better symmetry and lack of depression. This outcome indicates that reduction using a transconjunctival approach is a precise and accurate method of reduction compared with other existing methods in which, with the exception of open reduction, direct visualization of the fracture site is impossible and reduction has to be performed while the surgeon feels the fracture site with his/her hand.

However, in spite of the positive outcomes, our study has some limitations. Due to the anatomical structure of the nasal bone, the method we have introduced is particularly useful for fractures of the lateral nasal wall, and severe fractures or fractures that are difficult to access surgically, such as nasal bone tip fractures, after reduction using a transconjunctival approach, will further require conventional open or closed reduction and fixation using a Kirschner wire or miniplate and screws [4].

In conclusion, the major advantages of the method presented herein are that it enables simultaneous surgeries for nasal fracture accompanied by orbital blowout fracture, rather than treating the two fractures separately. Furthermore, it allows precise reduction of the nasal fracture by direct visualization of the fracture site without any additional incisions or difficult surgical techniques. In addition, by preventing the use of excessive force during reduction, this method can minimize damage to the nasal mucosa, thereby reducing the incidence of nasal bleeding. Finally, this method can be considered efficient because it does not require any additional specific equipment.

\section{CONFLICT OF INTEREST}

No potential conflict of interest relevant to this article was reported.

\section{PATIENT CONSENT}

The patients provided written informed consent for the publication and the use of their images.

\section{REFERENCES}

1. Rohrich RJ, Adams WP Jr. Nasal fracture management: minimizing secondary nasal deformities. Plast Reconstr Surg 2000;106:266-73.

2. Kim HS, Suh HW, Ha KY, Kim BY, Kim TY. The usefulness of the endonasal incisional approach for the treatment of nasal bone fracture. Arch Plast Surg 2012;39:209-15.

3. Yan Z, Zhou Z, Song X. Nasal endoscopy-assisted reconstruction of orbital floor blowout fractures using temporal fascia grafting. J Oral Maxillofac Surg 2012;70:1119-22.

4. Burm JS, Oh SJ. Indirect open reduction through intercartilaginous incision and intranasal Kirschner wire splinting of comminuted nasal fractures. Plast Reconstr Surg 1998;102:342-9.

5. Kim JH, Lee JH, Hong SM, Park CH. Open reduction of nasal bone fractures through an intercartilaginous incision. Acta Otolaryngol 2013;133:77-81

6. Ondik MP, Lipinski L, Dezfoli S, Fedok FG. The treatment of nasal fractures: a changing paradigm. Arch Facial Plast Surg 2009;11:296302.

7. Oh HK, Park YJ, Kim HS, Ryu JY, Kook MS, Park HJ, et al. A recent 5-year retrospective study on nasal bone fracture. J Korean Oral Maxillofac Surg 2008;34:230-6.

8. Santosh BS, Giraddi G. Transconjunctival preseptal approach for orbital floor and infraorbital rim fracture. J Maxillofac Oral Surg 2011;10:301-5.

9. Choi MS, Kim W, Youn S, Lee JH. Concomitant open reduction of a nasal bone fracture combined with a zygomatic fracture through a subciliary incision. J Craniofac Surg 2012;23:e25-7.

10. El-Anwar MW, Elsheikh E, Hussein AM, Tantawy AA, Abdelbaki YM. Transconjunctival versus subciliary approach to the infraorbital margin for open reduction of zygomaticomaxillary complex fractures: a randomized feasibility study. Oral Maxillofac Surg 2017;21:187-92. 